\title{
Systemic LP-PLA-2 cardiovascular marker response to direct medication delivery periodontal treatment
}

\author{
Duane C. Keller \\ Correspondence: drdkeller@drduanekeller.com \\ CrossMark \\ $\leftarrow$ Click for updates
}

St Louis Health and Wellness Keller Professional Group, St Louis, USA.

\begin{abstract}
Periodontal disease has been associated as a co-contributor for cardiovascular disease. Inflammatory markers such as lipoprotein-associated phospholipase A2 (Lp-PLA2) demonstrate an increased risk of heart attack and ischemic stroke. It is not known to what extent treating periodontal disease may affect changes in Lp-PLA2 levels. This case report discussed treating patients periodontal conditions with the Perio Protect Method (PPM) and evaluating systemic Lp-PLA2 levels, before, during and after periodontal treatment. This pilot study demonstrates that treating the patient's periodontal conditions resulted in decreasing the periodontal markers and lowering the Lp-PLA2 levels and these findings may be important as an adjunct of cardiovascular treatment.
\end{abstract}

Keywords: Periodontal disease, Lp-PLA2, cardiovascular disease, perio tray, perio protect method

\section{Introduction}

Measuring Lp-PLA2 blood levels evaluates a vascular specific inflammatory enzyme that participates in atherosclerotic plaque formation and plaque rupture and has been associated with a significant increased risk for heart attack and stroke if the Lp-PLA2 levels were greater than $200 \mathrm{ng} / \mathrm{ml}$ [1]. Lipoproteinassociated phospholipase $\mathrm{A} 2$ has been found to be associated with periodontal inflammation, indicating that periodontal treatments could reduce the risk of cardiovascular diseases. The recognition of this association has led investigators to determine Lp-PLA2 levels decrease following periodontal surgical and phase I periodontal therapy [2].

A pilot study was undertaken to determine if treating a patients periodontal disease with the Perio Protect Method might affect their systemic Lp-PLA2 levels. The Perio Protect Method uses a prescription tray (Perio Tray ${ }^{\circledR}$, Perio Protect LLC, St. Louis, MO www.perioprotect.com) to deliver medications into shallow $(<5 \mathrm{~mm})$ and deep $(>5 \mathrm{~mm})$ pockets in combination with mechanical debridement, scaling and surgery if required [3]. The Method clearly improves periodontal outcomes. A six month study of patients with moderate to severe periodontal disease using a 1.7 percent hydrogen peroxide gel (Perio gel ${ }^{\circledR}$, QNT Anderson LLC, Bismarck, ND) in a prescription customized tray (Perio Tray ${ }^{\circledR}$ ) as an adjunct to scaling and root planning (SRP) demonstrated over six months there was a clinically significant improvement in pocket depths and bleeding indices when compared to SRP alone, and it appears that the effect was not transitory. The test group reductions from baseline were maintained for six months [4]. This pilot study evaluates if this approach to periodontal disease treatment will also reduce LPPLA2 levels and benefit patients with cardiovascular disease.

\section{Methods}

Increased blood levels of Lp-PLA2 have been related to an increased risk of cerebral thrombosis [5] initial [6] and recurrent [7] coronary events, poor outcomes after acute coronary events [8] and cardiovascular disease associated with metabolic syndrome [9]. There is some controversy regarding the possible affect Lp-PLA2 may have on reducing atherogenesis by decreasing oxidized low-density lipoproteins (oxLDL) and platelet-activating factor (PAF), as degradation of these molecules may also have proinflammatory, proliferative and proatherogenic properties $[10,11]$. Because Lp-PLA2 levels relate to an increased risk to the patient, it would appear that reducing Lp-PLA2levels would be a beneficial goal of cardiovascular therapy [12].

Lp-PLA2 levels have been found to be elevated in chronic periodontal patients when compared to healthy control subjects [13]. Research from this study positively associated serum LpPLA2 levels and bleeding upon probing and periodontal inflammation prompting the question whether periodontal disease treatment could reduce the risk of cardiovascular disease in chronic periodontal patients. 
Duane C. Keller, Cardiovascular System 2014,

This pilot study is an attempt to answer the question if treating periodontal disease with the Perio Protect Method can reduce patient's Lp-PLA2 systemic inflammatory markers. The Perio Protect Method ${ }^{\circledR}$ (PPM) combines SRP and/or surgery with the use of a custom formed medical device (Perio Tray ${ }^{\circledR}$ ), to deliver doctor selected medications into the gingival sulcus or periodontal pocket. The tray delivery is used by patients at home under the dentist prescription in accordance with the periodontal conditions and treatments are modified as healing occurs.

The medications used in the pilot study were $1.7 \%$ hydrogen peroxide gel $\left(\right.$ Perio Ge ${ }^{\circledR}$ ) and 1-3 drops/tray Vibramycin Syrup [50 $\mathrm{mg} / 5 \mathrm{ml}$ doxycycline]. The medications areplaced by the patient into the Perio Tray and delivered into the periodontal pocket in accordance with the scope and magnitude of the patient's periodontal disease. The Perio Protect Protocol was determined by the patient deepest periodontal pockets as follows:

$$
\begin{array}{cc}
>6 \mathrm{~mm} \text { pockets } & 4 \text { times/day at } 15 \text { minutes } \\
3 \mathrm{~mm}-6 \mathrm{~mm} \text { pockets } & 3 \text { times/day at } 15 \text { minute } \\
<3 \mathrm{~mm} \text { pockets } & \text { (maintenance) } \\
& 2 \text { times/day at } 15 \text { minutes }
\end{array}
$$

The Silver Staff Method [14] of evaluation was used to measure the patient's Lp-PLA2 levels. The Silver Staff Method involved having the patient warm the patient's hand in warm tap water for 10 minutes. A finger was then cleansed with alcohol and a finger stick was performed at the edge of the fingerprint whorl. The patient's name and identification number were placed on the collection card. Drops of blood were then placed on the collection card until sufficient blood was accumulated to pass a registration line. The card was then processed as per the manufacturer's instructions and the card/sample was then mailed to Silver Staff for evaluation. The Silver Staff evaluators were blinded to the samples and testing as they only evaluated the blood sample results.

Six patients without experiencing a cardiovascular event were accepted into this case study. Each of the patients had active periodontal disease and agreed to have their Lp-PLA2 levels evaluated at onset, at one and three months. The acceptance criteria were the patient could not be under active cardiovascular treatment and could not take any cardiovascular or oral antibiotic medications during the course of the three month pilot study.

The patients started their three month periodontal disease treatment with Perio Tray administration of $<1 \mathrm{gm} 1.7 \%$ hydrogen peroxide and one to three drops Vibramycin Syrup/ tray. Following two weeks of Perio Tray usage scaling and root planning and/or debridement were completed. One of the six patients also received site specific surgery as needed. The patients were re-examined at one month for changes in their periodontal conditions and the blood samples were again taken and measured with the Silver Staff Method to evaluate their Lp-PLA2 levels. Alterations in the frequency of Perio Tray delivery were made in accordance with the treatment progress and/or new trays were fabricated as required by the healing that occurred.

The initial results were maintained by using the Perio Tray deliver of both medicaments one to two times a day for the remaining 3 month study period. At the three month visit, the periodontal conditions and blood samples were taken to evaluate the patient's Lp-PLA2 levels. The patients completed the three month case study without any complications or adverse situations.

\section{Results}

A total of 6 subjects completed the 3 month trial. After 4 weeks of periodontal treatment, analysis of deeper pockets $(>5 \mathrm{~mm}$ at baseline) showed that pocket probing depth (PPD) decreased from baseline by more than $0.90 \mathrm{~mm}$. At three months mean PPD decreases were $1.70 \mathrm{~mm}$ for the pilot study group. Analysis of shallower pockets (i.e., $\leq 5 \mathrm{~mm}$ at baseline) showed $0.57 \mathrm{~mm}$ reduction at one month and $1.08 \mathrm{~mm}$ reduction at three months.

Bleeding upon probing (BOP) was recorded as the number of bleeding sites recorded after probing the mesial buccal, buccal and distal buccal, mesial lingual, lingual and distal lingual aspects of each tooth. The reduction in bleeding for the test group showed a clinical improvement for both deep and shallow pockets (Figure 2). The mean BOP reductions for PPDs $>5 \mathrm{~mm}$ was approximately $62 \%$ at one month and an $80 \%$ decrease at three months. For PPDs in the $<5 \mathrm{~mm}$ range, there was a $74 \%$ decrease in bleeding upon probing at one month and almost a $90 \%$ decrease in the number of bleeding points at three months (Figure 3). The data indicate that prescription tray delivery of $1.7 \%$ hydrogen peroxide gel and Vibramycin resulted in a greater reduction of bleeding points in shallow pockets.

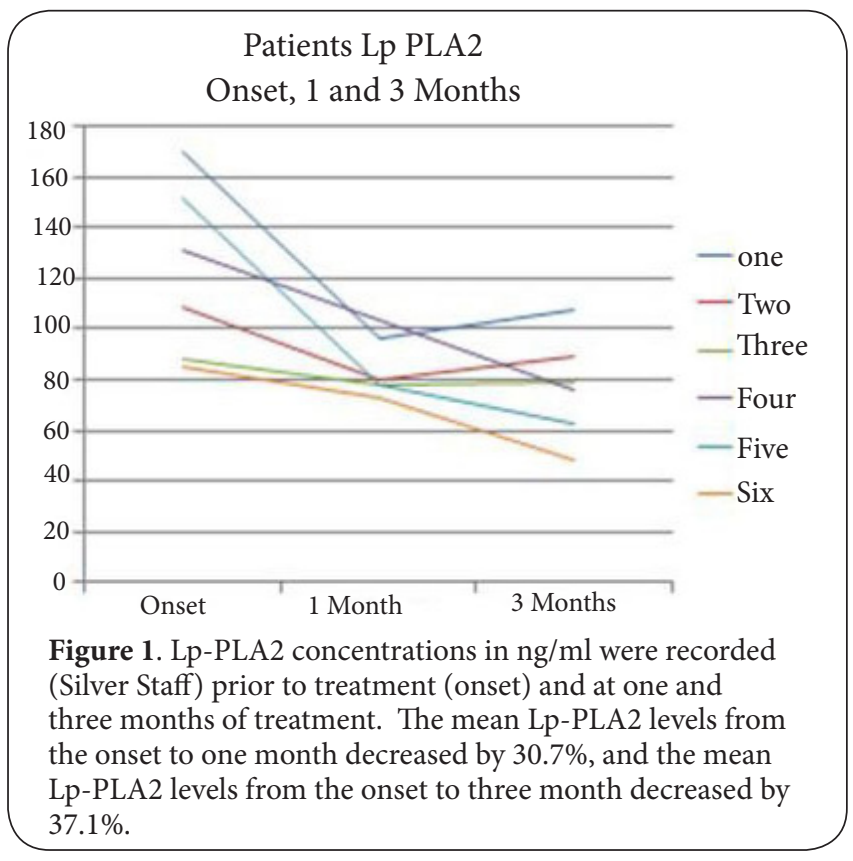



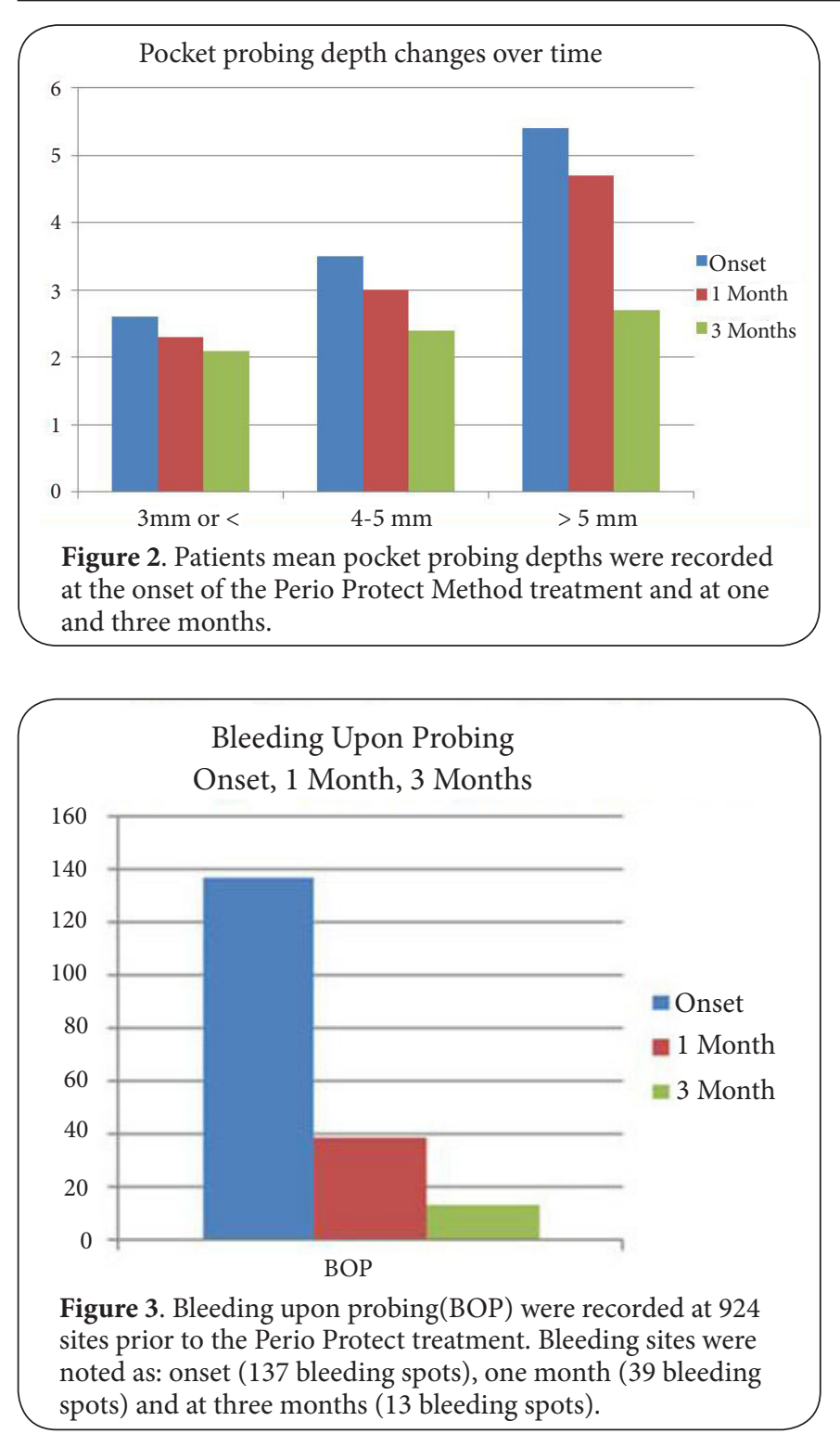

Patient's Lp-PLA2 levels were measured as $\mathrm{ng} / \mathrm{mL}$ prior to treatment and at one and three months of periodontal treatment. The mean Lp-PLA2 levels before treatment were $120.5 \mathrm{ng} / \mathrm{ml}$. The one month Lp-PLA2 levels were $80.7 \mathrm{ng} / \mathrm{ml}$ and the three month levels were $76.8 \mathrm{mg} / \mathrm{m}$. This equates to a $30.7 \%$ decrease in Lp-PLA2 levels at one month and a $37.1 \%$ decrease in LpPLA2 levels at three months. Individual patient results are indicated in Figure 1.

\section{Discussion}

The effectiveness of periodontal disease treatment using the Perio Protect Method 's combination of mechanical therapy with prescription tray delivery of medications was evaluated in subjects with chronic periodontitis to determine if treating their periodontal conditions would affect their systemic LpPLA2 inflammatory markers. The results demonstrated the periodontal treatment was effective in reducing pocket depths and bleeding upon probing (Figures 2 and 3 ) corroborating the findings of earlier trials $[4,15,16]$. The results also show reductions in baseline Lp-PLA2 scores for each patient.

It was hypothesized that treating periodontal disease would decrease patients Lp-PLA2 levels. Patient's Lp-PLA2 levels were evaluated at the onset of the study and at one and three months of using the Perio Protect Method with the custom formed Perio Tray. The mean Lp-PLA2 level prior to treatment was found to be a mean of $120.5 \mathrm{ng} / \mathrm{ml}$. The Lp-PLA2 levels decreased to a mean of $76.8 \mathrm{ng}$ at one month and further decreased to a mean of $80.7 \mathrm{ng}$ at three months. This equates to a $30.7 \%$ reduction in Lp-PLA2 levels in one month and a $37.1 \%$ reduction by the third month (Figure 1 ).

The only treatments rendered during the course of this study were dental procedures using the Perio Protect Method to address the patient's periodontal conditions. All patients were compliant in their periodontal treatments. Improvements in the patient's periodontal conditions (pocket probing depth and bleeding upon probing) appeared to coincide with patient's improvements in Lp-PLA2 levels, but the pilot study involved only 6 patients, which is not a significant number of patients for statistically significant findings to be determined.

\section{Conclusion}

This pilot study reports on unique findings where six patients with periodontal disease were treated with the Perio Protect Method and their Lp-PLA2 levels improved as their periodontal conditions improved. The results of this study appear to support the hypothesis that a relationship exists between periodontal disease and patient's systemic inflammatory Lp-PLA2 levels and that treating the periodontal disease may correlate with improved patients Lp-PLA2 levels. Further investigations will be required to determine the significance of this pilot program and if long-term management of periodontal disease will positively affect long-term Lp-PLA2 levels and/or cardiovascular health.

\section{Competing interests}

The author declares that he is the CSO of Perio Protect.

\section{Acknowledgement}

The author would like to thank Dr. Tanya Dunlap, Amy Doneen ARNP and Dr. Brad Bale for their support.

\section{Publication history}

Editor: Efstathios Vassiliadis, University of Copenhagen, Denmark. Received: 05-Sep-2014 Final Revised: 01-Nov-2014 Accepted: 07-Nov-2014 Published: 15-Nov-2014

\section{References}

1. Davidson MH, Corson MA, Alberts MJ, Anderson JL, Gorelick PB, Jones PH, Lerman A, McConnell JP and Weintraub HS. Consensus panel recommendation for incorporating lipoprotein-associated phospholipase A2 testing into cardiovascular disease risk assessment guidelines. Am J Cardiol. 2008; 101:51F-57F. | Article | PubMed

2. Keles GC, Cetinkaya BO, Pamuk F and Balli U. Activity of platelet 
Duane C. Keller, Cardiovascular System 2014,

http://www.hoajonline.com/journals/pdf/2052-4358-2-8.pdf

activating factor acetylhydrolase following phase I periodontal therapy. West Indian Med J. 2013; 62:62-7. I PubMed

3. Schaudinn C, Gorur A, Keller D, Sedghizadeh PP and Costerton JW. Periodontitis: an archetypical biofilm disease. J Am Dent Assoc. 2009; 140:978-86. | Article | PubMed

4. Putt MS and Proskin HM. Custom tray application of peroxide gel as an adjunct to scaling and root planing in the treatment of periodontitis: results of a randomized controlled trial after six months. J Clin Dent. 2013; 24:100-7. | PubMed

5. Ballantyne $\mathrm{CM}$, Hoogeveen RC, Bang $\mathrm{H}$, Coresh J, Folsom AR, Chambless LE, Myerson M, Wu KK, Sharrett AR and Boerwinkle E. Lipoproteinassociated phospholipase A2, high-sensitivity C-reactive protein, and risk for incident ischemic stroke in middle-aged men and women in the Atherosclerosis Risk in Communities (ARIC) study. Arch Intern Med. 2005; 165:2479-84. | Article | PubMed

6. Koenig W, Khuseyinova N, Lowel H, Trischler $\mathrm{G}$ and Meisinger C. Lipoprotein-associated phospholipase A2 adds to risk prediction of incident coronary events by C-reactive protein in apparently healthy middle-aged men from the general population: results from the 14-year follow-up of a large cohort from southern Germany. Circulation. 2004; 110:1903-8. | Article | PubMed

7. Koenig $W$, Twardella D, Brenner $H$ and Rothenbacher D. Lipoproteinassociated phospholipase A2 predicts future cardiovascular events in patients with coronary heart disease independently of traditional risk factors, markers of inflammation, renal function, and hemodynamic stress. Arterioscler Thromb Vasc Biol. 2006; 26:1586-93. | Article | PubMed

8. Möckel M, Müller R, Vollert JO, et al. Lipoprotein-associated phospholipase A2 for early risk stratification in patients with suspected acute coronary syndrome: a multi-parameter approach. Clin Res Cardiol. 2007; 96:604-612. | Pdf

9. Persson M, Hedblad B, Nelson JJ and Berglund G. Elevated Lp-PLA2 levels add prognostic information to the metabolic syndrome on incidence of cardiovascular events among middle-aged nondiabetic subjects. Arterioscler Thromb Vasc Biol. 2007; 27:1411-6. | Article | PubMed

10. Stafforini DM. Biology of platelet-activating factor acetylhydrolase (PAFAH, lipoprotein associated phospholipase A2). Cardiovasc Drugs Ther. 2009; 23:73-83. | Article | PubMed

11. Burke JE and Dennis EA. Phospholipase A2 biochemistry. CardiovasC Drugs Ther. 2009; 23:49-59. | Article | PubMed Abstract | PubMed Full $\underline{\text { Text }}$

12. Reddy KJ, Singh M, Bangit JR and Batsell RR. The role of lipoproteinassociated phospholipase $\mathbf{A} 2$ on cardiovascular disease risk assessment and plaque rupture: a clinical review. J Clin Lipidol. 2009; 3:85-93. | Article | PubMed

13. Zhou SY, Xiao WM and Ouyang XY. Lipoprotein-associated phospholipase A2 and serum lipid levels in subjects with chronic periodontitis and hyperlipidemia. Chin J Dent Res. 2012; 15:25-9. | PubMed

14. SilverStaff Clinical laboratories. | Website

15. Keller D. Managing periodontal disease in a patient suffering from renal failure. Dent Today. 2008; 27:144-7. | PubMed

16. Keller D. Management of periodontitis for HIV-AIDS patients: case study. Dent Today. 2006; 25:112-3. | PubMed

\section{Citation:}

Keller DC. Systemic Lp-PLA-2 cardiovascular marker response to direct medication delivery periodontal treatment. Cardio Vasc Syst. 2014; 2:8. http://dx.doi.org/10.7243/2052-4358-2-8 